\title{
Application of a Mobile Chronic Disease Health-Care System for Hypertension Based on Big Data Platforms
}

\author{
Dingkun Li $\left(\mathbb{D}^{1},{ }^{1}\right.$ Hyun Woo Park, ${ }^{1}$ Erdenebileg Batbaatar, ${ }^{1}$ Lkhagvadorj Munkhdalai, \\ Ibrahim Musa, ${ }^{1}$ Meijing Li, ${ }^{2}$ and Keun Ho Ryu $\mathbb{D}^{1}$ \\ ${ }^{1}$ Database/Bioinformatics Lab, School of Electrical \& Computer Engineering, Chungbuk National University, \\ Cheongju 28644, Republic of Korea \\ ${ }^{2}$ College of Information Engineering, Shanghai Maritime University, Shanghai 201306, China
}

Correspondence should be addressed to Keun Ho Ryu; khryu@chungbuk.ac.kr

Received 15 February 2018; Accepted 26 March 2018; Published 29 May 2018

Academic Editor: Ka L. Man

Copyright (C) 2018 Dingkun Li et al. This is an open access article distributed under the Creative Commons Attribution License, which permits unrestricted use, distribution, and reproduction in any medium, provided the original work is properly cited.

\begin{abstract}
Hadoop is a globally famous framework for big data processing. Data mining (DM) is the key technique for the discovery of the useful information from massive datasets. In our work, we take advantage of both platforms to design a real-time and intelligent mobile health-care system for chronic disease detection based on IoT device data, government-provided public data and user input data. The purpose of our work is the provision of a practical assistant system for self-based patient health care, as well as the design of a complementary system for patient disease diagnosis. This system was only applied to hypertensive disease during the first research stage. Nevertheless, a detailed design, an implementation, a clear overview of the whole system, and a significant guide for further work are provided; the entire step-by-step procedure is depicted. The experiment results show a relatively high accuracy.
\end{abstract}

\section{Introduction}

Hypertension is a condition in which a person's blood pressure is above normal or optimal limit of $120 \mathrm{mmHg}$ for systolic pressure and $80 \mathrm{mmHg}$ for diastolic pressure. Increased blood pressure in the long term can lead to conditions that could threaten the health of the sufferer. Several conditions can cause disturbances in hypertensive cardiovascular organs such as stroke and heart failure, so sometimes mentioned that hypertension is a silent killer, because sufferers sometimes do not realize that he was exposed to conditions of hypertension [1]. The classification of blood pressure in adult divided into 4 classes which have been shown in Table 1.

Nevertheless, in our work, we treat prehypertension, HP stage 1, and HP stage 2 as hypertension with no difference.

Hadoop, which is based on MapReduce, has been one of the most important and popular techniques in the field of big data analysis during the last few years. Undoubtedly, it is the key technique for massive data analysis. Alternatively, Spark is a promising distributed framework that runs memory data on clusters at a speed that is considerably faster than that of Hadoop. Data mining (DM) is the key technique for the discovery of the useful information from well-processed data at the intersection of areas such as machine learning, statistics, and database systems. In the present work, the aim is the exploitation of the use of Hadoop, Spark, and DM techniques to provide a more powerful way of handling big data at high extents of speed, safety, and accuracy.

In recent years, the Hadoop framework has been widely used for the delivery of health care as a service [2]; moreover, a wide variety of organizations and researchers have used Hadoop for health-care services and clinical-research projects [3]. Taylor provided a detailed introduction on the use of Hadoop in bioinformatics [4], while Schatz developed an operations support system (OSS) package named CloudBurst 
Table 1: Classification of Blood Pressure.

\begin{tabular}{lcc}
\hline Classification & Systolic $(\mathrm{mmHg})$ & Diastolic $(\mathrm{mmHg})$ \\
\hline Normal & $<120$ & And $<80$ \\
Prehypertension & $120 \sim 139$ & Or $80 \sim 89$ \\
HP Stage 1 & $140 \sim 149$ & Or $90 \sim 99$ \\
HP Stage 2 & $\geq 150$ & Or $\geq 100$ \\
\hline
\end{tabular}

that provides an algorithmic parallelization model for which Hadoop MapReduce is used [5]. Indeed, the Hadoop framework has been employed in numerous important works to provide major contributions to the health-care field. The other big data processing framework, Spark, leverages a synergistic combination of the smartphone and the smartwatch in the monitoring of multidimensional symptoms such as facial tremors, dysfunctional speech, limb dyskinesia, and gait abnormalities [6].

Over many years, a large amount of health-care research work has been completed using DM techniques. In $[7,8]$, the authors used classification and regression techniques to predict conditions like cardiovascular disease and heart disease. In $[9,10]$, integrated DM techniques are provided for the detection of chronic and physical diseases. Further, a number of other research works, like $[11,12]$, used the advantages of DM to develop new methodologies and frameworks for health-care purposes.

The major goal of health-informatics research is the improvement of the quality and the cost of care that are provided to users, or the health-care output [13]. The purpose of the present work is the exploitation of Hadoop, Spark, and DM techniques for the design of a comprehensive, real-time, and intelligent mobile health-care system for chronic disease detection and prediction. The system is designed to provide an assistant system for self-based user health care, as well as a complementary system for the daily diagnostic work of doctors.

A series of challenges arise in the development of a big data-based health-care system. Firstly, it is extremely difficult to obtain high-quality and relevant medical data. One reason for this is that hospitals or the patients themselves are not willing to offer personal data for public research due to privacy policies. Another reason is the need to engage with a variety of data sources, such as the collection of data from hospitals, health-care centers, governments, laboratories, and the patients' families, which can cause serious missingdata problems. For instance, only the hospital-treatment data of patient $\mathrm{A}$ are available while the lifestyle (smoking, drinking, etc.) data are missing, and only the lifestyle data of patient B are available while the patient's treatment data are missing. The work of [14] confirms this varied-source characterization of health-care data collection and the complexity of different data forms. Secondly, data analysis is a challenging work. Even though a great quantity of research work has been completed to process and analyse data, a high-quality framework with highly precise predictive and analytic results is still mostly elusive [1]. Thirdly, the difficulty regarding the creation of a tool that can break the borders between the patient, health-care providers, and public health-care organizations to connect these parties in a practically meaningful manner is another obstacle [15].

The contributions of the present work are as follows: (1) exploration of the possibility of the utilization of Hadoop, Spark, and DM techniques in the work regarding healthcare big data. (2) Depiction of a detailed step-by-step design of the health-care system for disease detection and prediction. (3) Provision of an overview for the next research stage and a guide for other similar systems. (4) Minimization of the monetary cost through the use of the Google Cloud services FCM and GCSql, which also guarantee real-time data transactions. A preliminary version of the present work has been reported in [15].

This paper is organized as follows: a description of the related work is provided in Section 2; an overview of the proposed system is introduced in Section 3; the design details are described in Section 4; the experiment results are described in Section 5; and Section 6 concludes this work and introduces future work.

\section{Selection Techniques and Algorithms}

This section briefly describes the related platforms, algorithms, and some of the key techniques that were used in the undertaking of the present work.

2.1. Hadoop, Spark, and Data Mining. Hadoop consists of the HDFS (Hadoop Distributed File System), HBase, and Hadoop MapReduce, making it very suitable for big data analyses [16]. As a 100\% open-source framework, it has been widely used in almost every field for big data processing. In the last few years, Apache Spark [17] received great attention in the big data and data science fields, mainly because of its easier, friendlier application program interface (API) and an enhanced memory management compared with MapReduce; therefore, developers could concentrate on the datacomputation logical operations rather than the background details of the computational execution.

It is difficult to find a coincident DM definition, but one of the widely accepted definitions states that DM is the process of discovering interesting patterns and knowledge from large amounts of data [18]. Its other close concept is called knowledge discovery in databases (KDD); DM is the analytical step of KDD. In this paper, a commonly used classification algorithm called C4.5 will be used for the disease-rule generation since it is simple, stable, and produces results of a relatively high accuracy.

2.2. C4.5. C4.5 is an algorithm that was developed by Ross Quinlan and is used to generate decision trees [18]. C4.5 is an extension of Quinlan's earlier ID3 algorithm. The decision trees that are generated by $\mathrm{C} 4.5$ can be used for the purpose of classification, and for this reason, C4.5 is often referred to as a statistical classifier.

In general, the steps of the $\mathrm{C} 4.5$ algorithm for the building of decision trees are as follows: choose the attribute for the root node; create the branch for each value of that attribute; split the case according to the branches; and repeat 


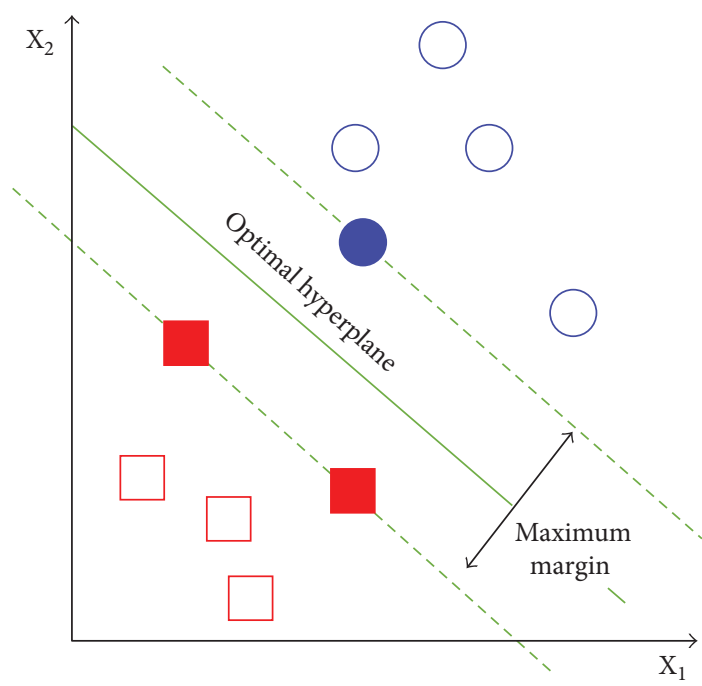

FIGURE 1: Maximum margin, the vectors on the dashed line are the support vectors.

the process for each branch until all of the branch cases are of the same class [18].

2.3. Support Vector Machine. Support vector machine (SVM) [19] has been used to select features and generate the classifier. For feature selection, this method is a backward sequential selection approach. One starts with all the features and removes one feature at a time until only $r$ features are left. The operation of the SVM algorithm is based on finding the hyperplane that gives the largest minimum distance to the training examples. The basic concept is described using Figure 1.

The strategy ranks the features according to their influence on the decision hyperplane.

The optimal hyperplane is used to classify the data into different classes in two or more dimensionalities.

2.4. Hybrid Feature Selection Mechanism. Feature selection aims at finding the most relevant features of a problem domain. Primarily, there are two kinds of feature selection methods, filters and wrappers. The filters work fast but its result is not always satisfactory. While the wrappers guarantee good results, they are very slow when applied to wide feature sets which contain hundreds or even thousands of features. According to work [20], a hybrid feature selection mechanism takes advantage of both filter and wrapper feature selection methods is used to improve the computation speed and accuracy.

Inspired by [20], we developed our feature selection mechanism. The architecture is show in Figure 2.

\section{Main Framework}

An overview of the whole system is given in this section, and this is followed by a description of the implementation details in Section 4. The proposed system comprises four modules. The overview of the architecture of the entire system is depicted in Figure 3 [15]. The four modules in the figure

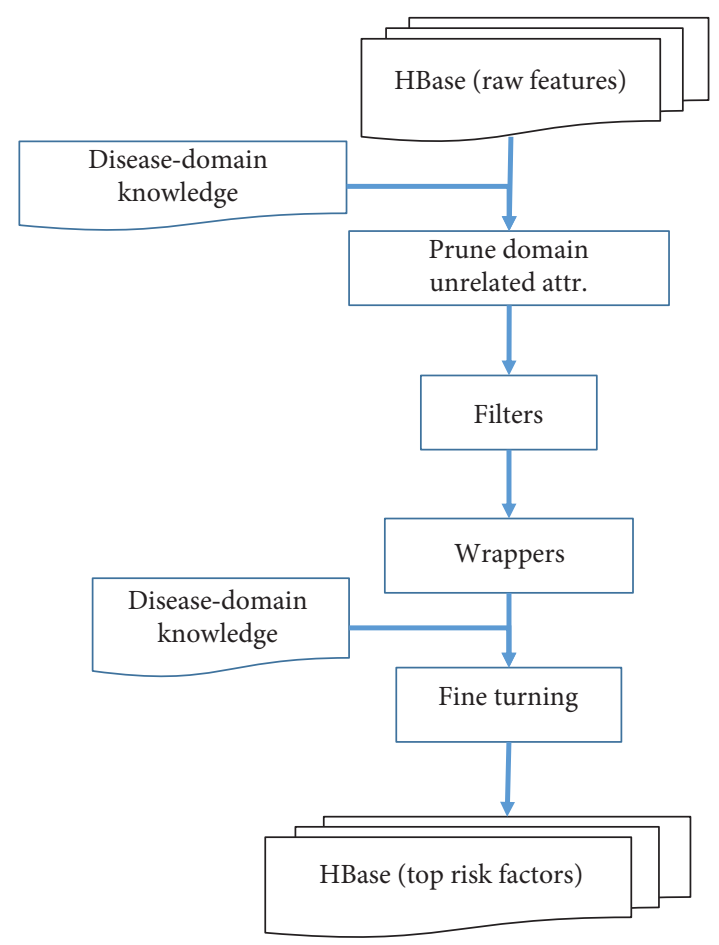

Figure 2: Risk factors (features) selection mechanism. We combined the expert domain knowledge for the purpose of pruning the unrelated attributes. Filter methods followed by wrapper methods are used select the features for disease rule generation. The evaluated result is stored in HBase.

are named as follows: (1) data collection module, (2) data storage module, (3) third-party-server (TPS) module, and (4) Cloud service module.

Module 1a is used to collect the streaming data and structured data by IoT devices such as Fitbit Charge 2, mobile phone sensors. $1 \mathrm{~b}$ is used to import structured, semistructured, and unstructured data from various data sources like hospitals, governments, families, user inputs, and so on. Besides, we have developed a mobile app to collect user input data such as lifestyle and food intake data.

Module 2 is used to store the data in HBase collected by module 1 . The data collected by the system is of three types: the structured, the semi-structured, and the unstructured data. Firstly, all these three kinds of data will be stored in HBase as it is quite suitable for mass data preprocessing and storage. Then this data should be converted into structured data for further processing.

Module 3 is used for the processing and analysis of the data based on the Hadoop/Spark cluster which is the key module of the whole system; all the data processing and analysis work will be done by this module. It is used for data statistical analysis, patient emergency detection, and disease prediction and detection. It also responses for message like data analysis results generation. These result will be sent to module 4.

Module 4 is used for the message dissemination. This model is implemented by using Google Cloud SQL (GCSql) and Google Firebase Cloud Messaging (GFCM) services. When receiving the requests from the TPS, Cloud model 


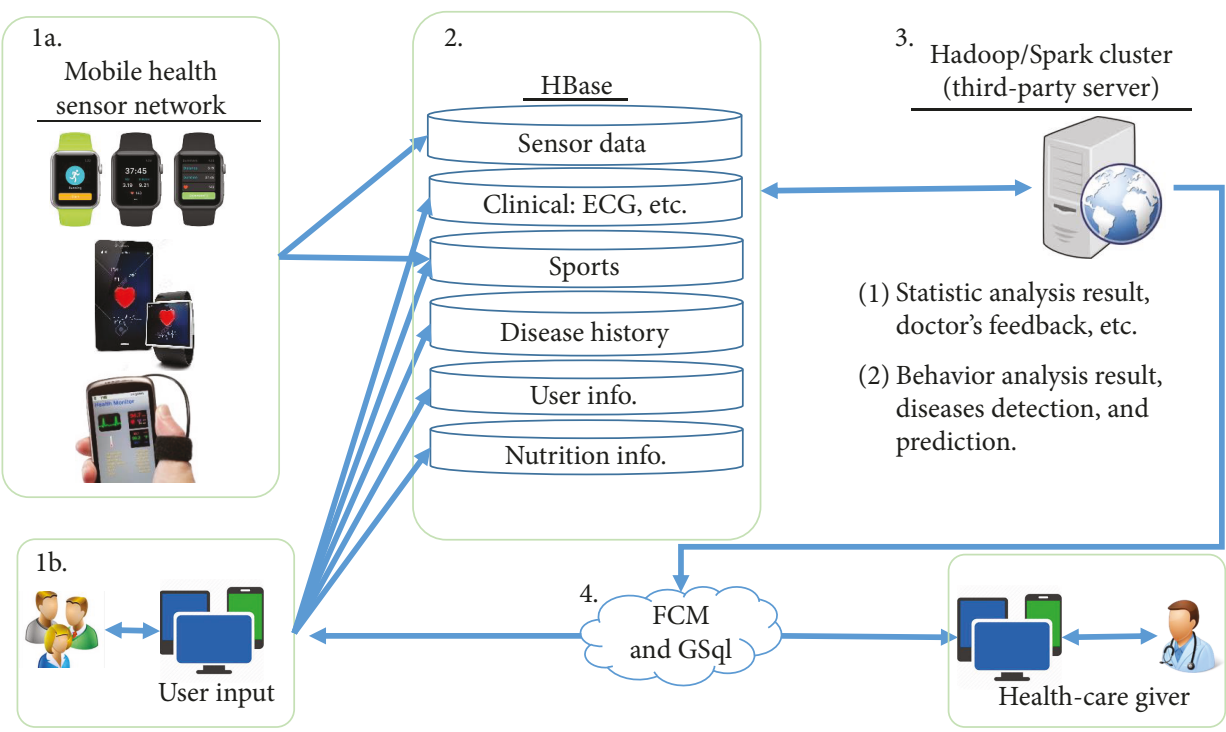

FIGURE 3: Systemic architecture: the four modules are marked 1 (1a and 1b), 2, 3, and 4.

responses immediately according to these requests, stores data, or sends data to the devices registered to it.

Further details have been given in a previous work of the authors of the present study [15].

\section{System Implementation Details}

In this section, descriptions of the systemic data flow, the data storage and processing, and the disease detection and prediction based on large medical datasets are provided.

4.1. Data Collection, Preprocessing, and Storage. To obtain high-quality structured datasets, database processing, natural language processing (NLP), and image-processing techniques are combined with the DM data-preprocessing techniques that are used by the TPS to process the different kinds of data (structured, semistructured, and unstructured), and the data are then transformed into a structured data record. The result is then stored in the HBase.

(1) For the structured dataset (mostly imported from the other public Web services) that includes patient information, prescriptions, and disease histories, it is relatively easier to import the data from the rational DB to the HBase using Sqoop [21].

(2) For the semistructured dataset, which includes HTML, XML, and Json documents, the TPS will design row keys like the d001 for the HBase table, including its document-information column value together with its family map that is called "column family" (it comprises the document timestamps of the HBase), as shown in Figure 4. The semistructured data will be converted to the structured data in the HBase, as shown in Figure 5.

(3) For the unstructured data, like clinic notes and the stream data from mobile sensors, they will be managed by the system in a particular way. The clinic

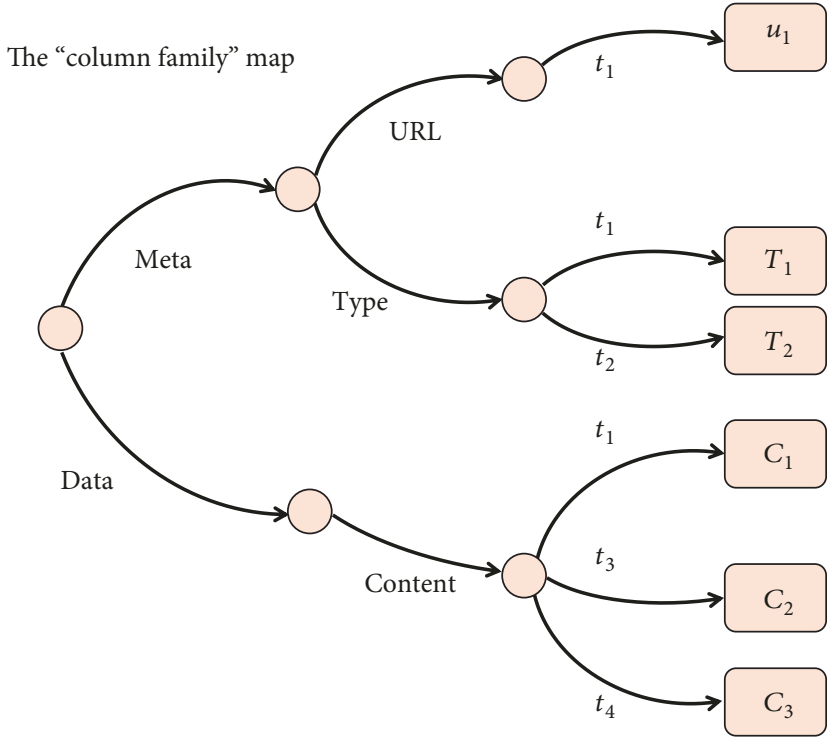

FIgURE 4: Column family map. The meta information and data content can be depicted by this map with their timestamp information. Where $u_{i}$ stands for document URL, $T_{i}$ stands for document type, and $\mathrm{C}_{\mathrm{i}}$ stands for document content.

notes contain a lot of the textual information, [22] providing an efficient way to convert this data into structured data through the use of NLP techniques, text-mining algorithms, and the MapReduce framework. The same strategy is used in the proposed system to deal with this problem, and the procedure is shown in Figure 6.

The stream data are handled using Apache Spark [23] techniques; the basic procedure is shown in Figure 7. Finally, the output will be stored in the HBase. After the preprocessing step, all kinds of data will be converted into structured data and stored in distributed HBase regional servers for further processing. 


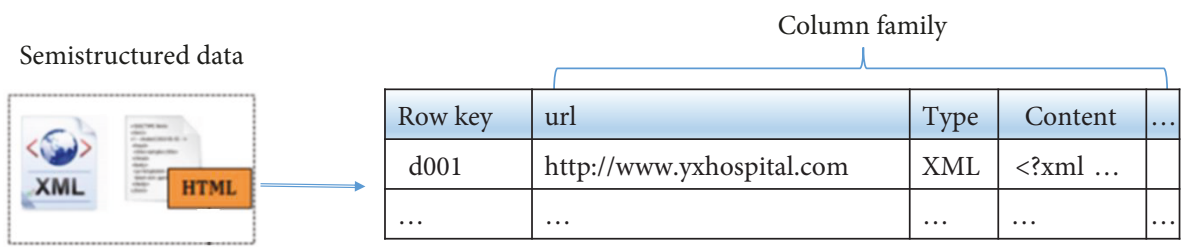

FIGURE 5: HBase example for semistructured data storage. Semistructured data (such as XML and HTML files) will be converted to structured data which has the similar structure as relational data base table.

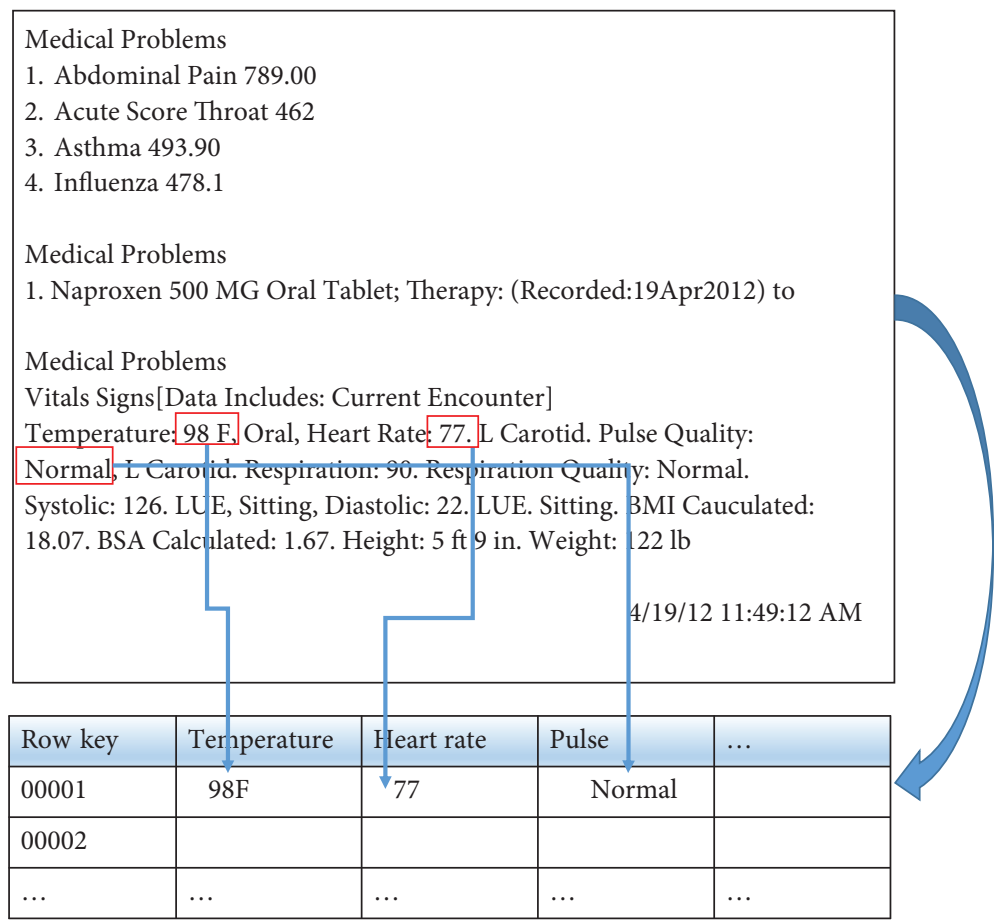

FIGURE 6: HBase example for unstructured data storage. Important information will be extracted from the raw data and stored in table format which is appropriate for further analysis.

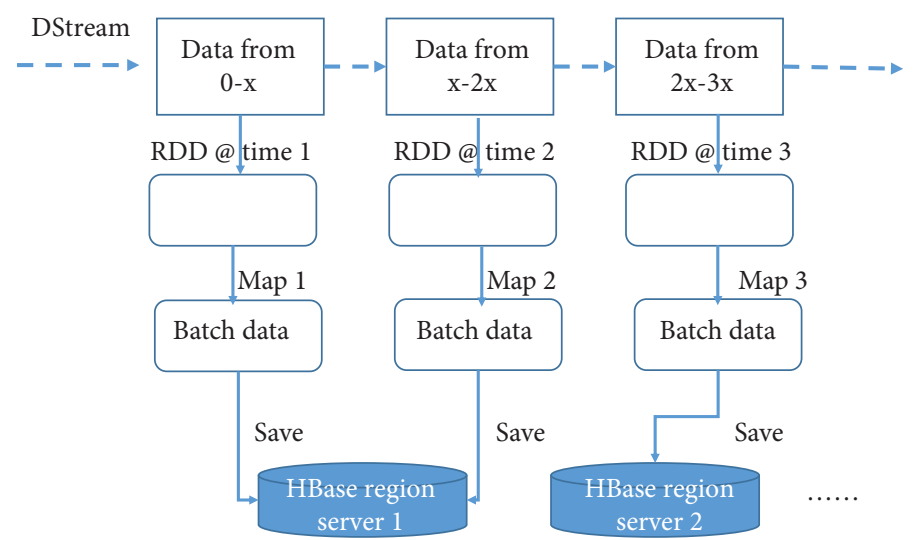

Figure 7: Streaming-data processing by Spark. The Spark engineer divides the stream data into sequential blocks and these blocks are stored in different nodes of the cluster.

4.2. Disease Data Statistical Analysis. Among the whole existing dataset, some of the patient data are treated as the training set for the disease-rule generation (some of the datasets are not disease related). The first step here is the counting of the diseases of the patients with their personal information, like their gender, age, nationality, and occupation. Since the data were stored in the HBase, the MapReduce framework was used to count the diseases. The training data 
Condition attribute value

Decision attribute value

\begin{tabular}{|c|c|c|c|c|c|c|}
\hline & & & & & & \\
\hline Row key & Temperature & Heart rate & Pulse & $\ldots$ & Disease & \\
\hline 00001 & $98 \mathrm{~F}$ & $77 \mathrm{~L}$ & Normal & & Hypertension & \\
\hline 00002 & $98.5 \mathrm{~F}$ & $80 \mathrm{~L}$ & Normal & & Diabetes I & $\begin{array}{c}\text { HBase region } \\
\text { server } 1\end{array}$ \\
\hline 00003 & $97 \mathrm{~F}$ & $70 \mathrm{~L}$ & Fast & & Diabetes II & \\
\hline 00001 & $99.5 \mathrm{~F}$ & $79 \mathrm{~L}$ & Normal & & Bradycardia & \\
\hline$\cdots$ & $\cdots$ & $\cdots$ & $\cdots$ & $\cdots$ & $\cdots$ & HBase region \\
\hline 00008 & & & & & Hypertension & \\
\hline$\cdots$ & $\cdots$ & $\cdots$ & $\cdots$ & $\cdots$ & $\cdots$ & \\
\hline
\end{tabular}

Figure 8: Training data example. Data comes from different sources and stored in different cluster nodes.

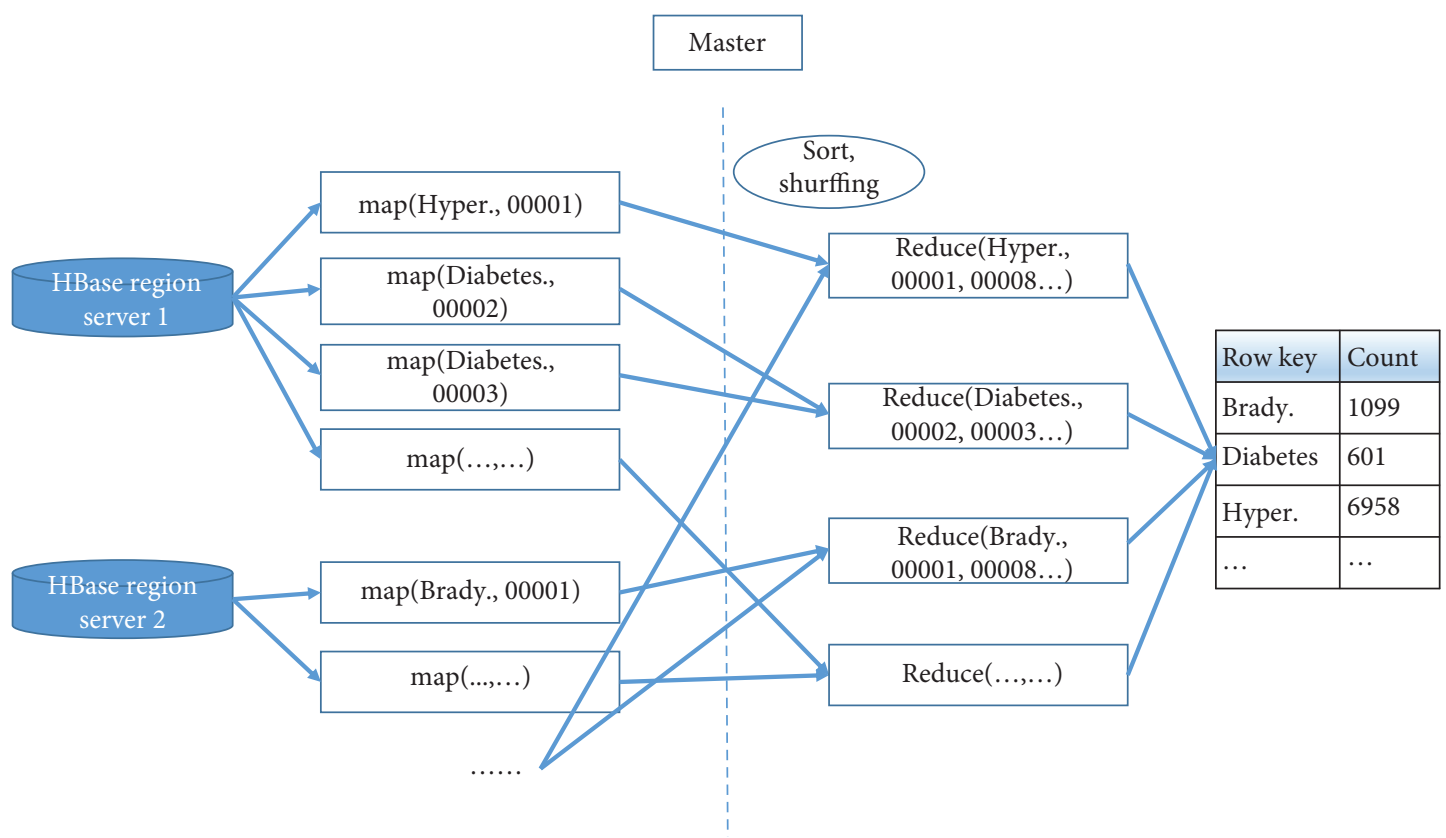

Figure 9: MapReduce procedure for disease count. Disease name is key and row ids are value for Map function. Disease name is key and all the row ids for the same disease are value for Reduce function. The count of row ids is the count for specified disease.

are stored in separate regional servers, as shown in Figure 8. The disease-count MapReduce procedure running in TPS is depicted in Figure 9.

The disease-count algorithm running in distributed environment is shown in Algorithm 1. It consists of two main procedures which are Map and Reduce. Map function is used to assign constant value 1 in terms of each row for distinct disease and patient. Reduce function is used to add all $1 \mathrm{~s}$ together for the same disease, the sum of $1 \mathrm{~s}$ is the count of the specified disease.

Based on the output, it is straightforward to obtain the patient list for a specified disease, as well as all of the personal information according to the patient ID.

4.3. Risk Factor Selection. The risk factor (RF) selection procedure is a process for feature selection. Hybrid feature selection has been applied to the raw dataset. First, we apply $t$-test

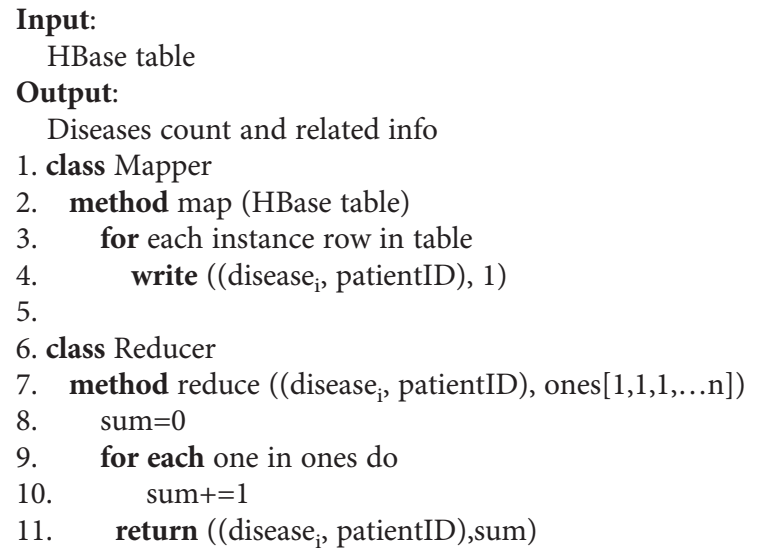

Algorithm 1: Disease count running on HBase. 


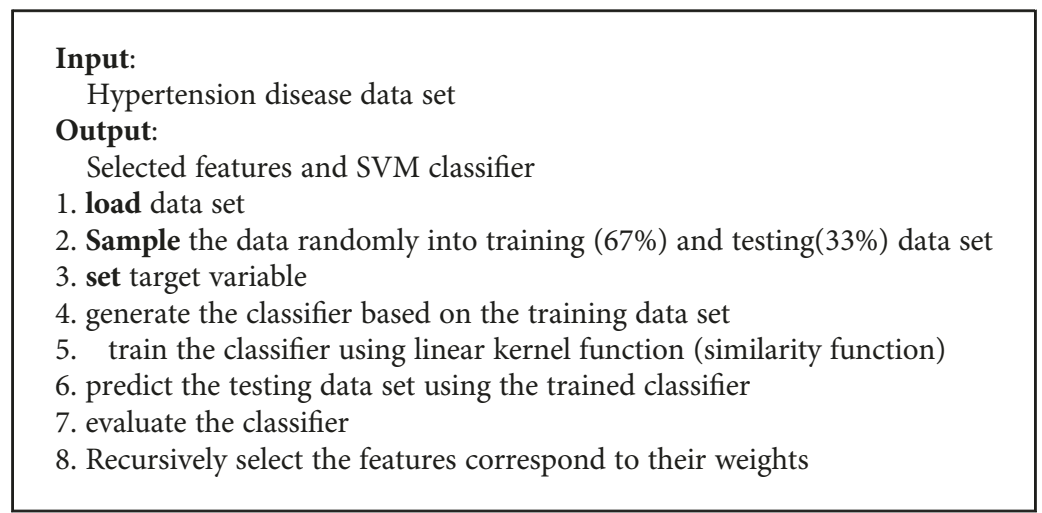

Algorithm 2: Linear SVM pseudocode.

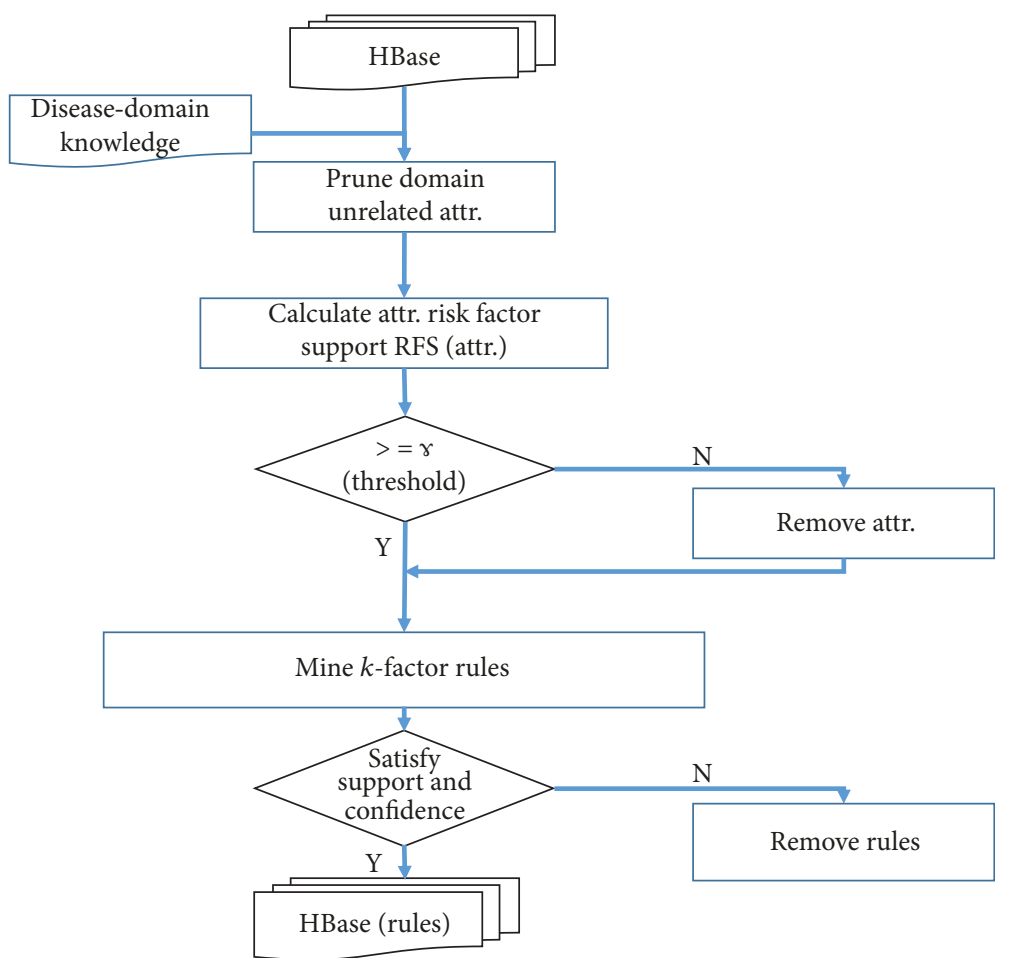

FIGURE 10: Disease-rule generation procedure. Domain expert's knowledge is used to prune the unrelated attributes for the first round. Then RFS is calculated for each remaining attributes to compare with predefined threshold $\gamma$, if larger than $\gamma$, it is treated as risk factor for further rule generation step.

combined with chi-squared test as filters to prune unrelated features according to research [11], chi-square and $t$-test are fast and can achieve relative high accuracy. The chisquared test formula is,

$$
\chi^{2}=\sum_{i=1}^{k} \frac{\left(x_{i-} m_{i}\right)^{2}}{m_{i}}
$$

where the expected numbers $m_{i}$ were large enough known numbers in all cells assuming every $x_{i}$ may be taken as normally distributed, and reached the result that, in the limit as $\mathrm{n}$ becoming large, $\chi^{2}$ followed the chi-squared distribution with (k-1) degrees of freedom.
The $t$-test can be used, for example, to determine if two sets of data are significantly different from each other. The $t$-test

$$
t=\frac{Z}{s}=\frac{(\bar{x}-\mu) /(\sigma / \sqrt{n})}{s},
$$

where $\bar{x}$ is the sample mean from a sample $\mathrm{X}_{1}, \mathrm{X}_{2}, \ldots, \mathrm{X}_{n}$, of size $n, s$ is the ratio of sample standard deviation over population standard deviation, $\sigma$ is the population standard deviation of the data, and $\mu$ is the population mean.

After the first step, we propose a wrapper method based on linear support vector machines (SVMs). Firstly, we train the linear SVM on a subset of training data and retain only 
Input:

Data partition D: a training set and associated class label C

Attribute list L (selected disease risk factors in previous step)

Output:

Decision tree with its root $\mathrm{N}$

Method:

1. Create a node $\mathrm{N}$,

2. if samples has the same class, $C$ then,

3. return $\mathrm{N}$ as leaf node with class $\mathrm{C}$ label

4. if list of attributes is empty then

5. return $\mathrm{N}$ as leaf node with class label that is the most class in training set.

6. Choose test factor, that has the most GainRatio using attribute_selection_method

7. give node $\mathrm{N}$ with test-attribute label

8. for each attribute $a_{i}$ in $L$

9. add branch in node $\mathrm{N}$ to test-attribute $=\mathrm{a}_{\mathrm{i}}$

10. make partition for sample $s_{\mathrm{i}}$ from training set where test-attribute $=\mathrm{a}_{\mathrm{i}}$

11. if $s_{\mathrm{i}}$ is empty then

12. attach leaf node with the most class in training set

13. else attach node that generated by Gnerate_decision _tree ( $s_{\mathrm{i}}$, L, test-attribute)

14. return $\mathrm{N}$

Algorithm 3: C4.5 for important disease rule generation.

TABLE 2: Disease-count results.

\begin{tabular}{lccccc}
\hline Disease & Hyperten. & Dyslip. & Ostarthritis. & Diabetes & Asthm. \\
\hline Count & 9385 & 3213 & 5223 & 3500 & 923 \\
\hline
\end{tabular}

TABLE 3: Statistical analysis.

\begin{tabular}{|c|c|c|c|}
\hline & Weighted $N(\%)$ & Women (\%) & Men (\%) \\
\hline \multicolumn{4}{|l|}{ Age } \\
\hline $20-44$ & $20,131(40.6)$ & $11,416(40.5)$ & $8715(41.5)$ \\
\hline $45-64$ & $17,623(35.6)$ & $9946(35.3)$ & $7677(36.6)$ \\
\hline $65+$ & $11,775(23.8)$ & $6822(24.2)$ & $4593(21.9)$ \\
\hline \multicolumn{4}{|l|}{ Marital status } \\
\hline Married & $42,478(86.6)$ & $24,747(88.5)$ & $17,731(84.1)$ \\
\hline Never married & $6569(13.4)$ & $3208(11.5)$ & $3361(15.9)$ \\
\hline \multicolumn{4}{|l|}{ Education } \\
\hline University & $12,990(28.9)$ & $6612(25.5)$ & $6378(33.6)$ \\
\hline High school & $14,876(33.1)$ & $8129(31.3)$ & $6747(35.6)$ \\
\hline Middle school & $4950(11.0)$ & $2659(10.2)$ & $2291(12.1)$ \\
\hline Elementary school & $12,125(27.0)$ & $8568(33.0)$ & $3557(18.8)$ \\
\hline \multicolumn{4}{|l|}{ Occupation } \\
\hline Office worker & $8993(20.1)$ & 4104 (15.8) & $4889(25.9)$ \\
\hline Manual worker & $17,181(38.4)$ & 8099 (31.3) & $9082(48.2)$ \\
\hline Unemployed & $18,602(41.5)$ & $13,710(52.9)$ & $4892(25.9)$ \\
\hline \multicolumn{4}{|l|}{ Income Level } \\
\hline 1st quartile & $12,077(24.8)$ & $6862(24.8)$ & $5215(24.9)$ \\
\hline 2nd quartile & $12,183(25.1)$ & $6940(25.1)$ & $5243(25.0)$ \\
\hline 3rd quartile & $12,193(25.1)$ & $6930(25.1)$ & $5263(25.1)$ \\
\hline 4th quartile & $12,167(25.0)$ & $6912(25.0)$ & $5255(25.1)$ \\
\hline \multicolumn{4}{|l|}{ Hypertension } \\
\hline No & 8619 (47.9) & $4954(47.4)$ & $3665(48.5)$ \\
\hline Yes & $9383(52.1)$ & $5488(52.6)$ & $3895(51.5)$ \\
\hline
\end{tabular}

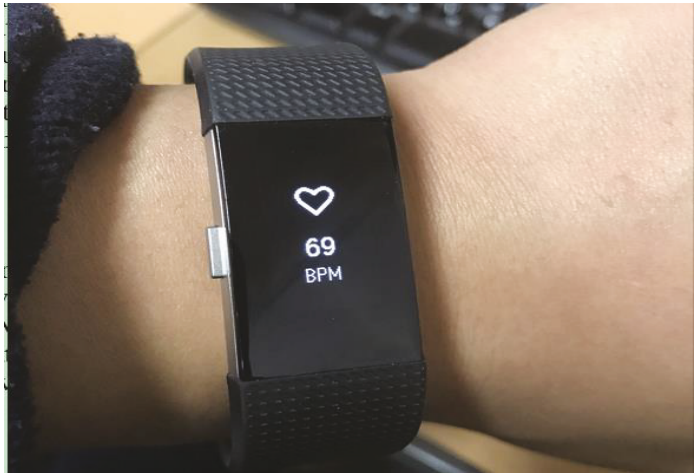

FIGURE 11: Screenshot of the IoT device we used in our system.

those features that correspond to highly weighted components (in absolute value sense) of the normal to the resulting hyperplane that separates positive and negative examples for the class. Secondly, recursively eliminate features whose weight value is close to zero. Finally, the features remained are selected as risk factor candidates. We created a representation result in experimental session, Chapter 5. The pseudocode is given in Algorithm 2 below. First, we divide the original dataset into training and testing dataset; the SVM classifier is generated based on this training dataset. After evaluating the classifier, the features will be recursively selected according to the weight until the stop criterion has been met.

The result will be described in experimental session.

4.4. Disease-Rule Generation. The format of disease rules is like that of the IF THEN rule; for example, IF (edu = elementary, $\mathrm{B} 1<=0.86 \mathrm{mg} /$ day, married), THEN (hypertensio$\mathrm{n}=$ yes). The purpose here is the mining of all of the disease-related rules from the training dataset for a further data analysis including disease prediction and disease 


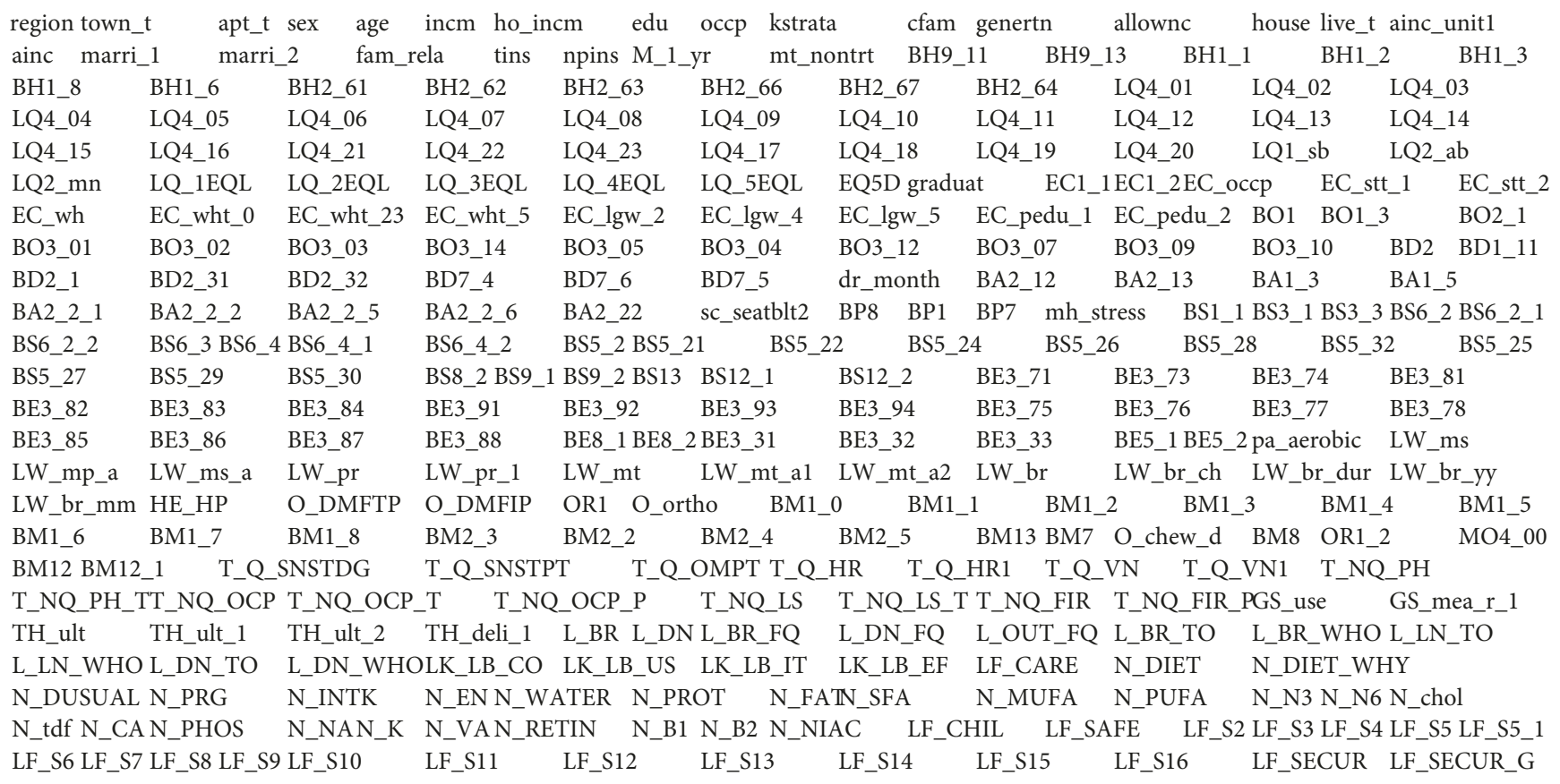

FIgURE 12: Features selected after filters methods: $t$-test and chi-squared test. This result is used as the input for wrapper method.

TABLE 4: SVM-based attribute risk factor computation.

\begin{tabular}{lcr}
\hline Number & Ranked risk factors & Weight \\
\hline 1 & Age & 0.0890 \\
2 & DE1_pr: current status of diabetes & 0.0885 \\
3 & DN1_dg: kidney failure diagnosis & 0.0881 \\
4 & BD1_11: (12 years old or older) frequency of drinking for 1 year & 0.0873 \\
5 & BP8: average sleep time per day & 0.0841 \\
6 & BP1: usually stressed & 0.0370 \\
7 & BS3_1: (adult) currently smoking & 0.0322 \\
8 & N_NA: sodium intake (mg) & 0.0321 \\
\hline
\end{tabular}

TABLE 5: Hypertension-disease rules generated by C4.5.

\begin{tabular}{|c|c|c|}
\hline Number & Rules & Conf. \\
\hline 1 & If (age $>70$, DE1_pr $=1)$ - >yes & 0.86 \\
\hline 2 & If $(46.5<$ age $<=70$, BD1_11>=6) $->$ yes & 0.74 \\
\hline 3 & If $\left(\mathrm{BP} 8>8, \mathrm{~N} \_\mathrm{B} 1<=0.88 \mathrm{mg}\right)->$ yes & 0.73 \\
\hline 4 & If (marri_1=2, BD1_11>=6) - >yes & 0.73 \\
\hline 5 & If $($ age $<=46.5$, BD1_11<4, N_WATER $>1060.5 \mathrm{ml} /$ day) $->$ no & 0.72 \\
\hline 6 & If (DN1_dg=1, marri_1=2) - >yes & 0.63 \\
\hline 7 & If (N_B1 < = 0.86 mg/day, N_WATER $<=485.63)->$ yes & 0.61 \\
\hline 8 & If (marri_1=1, N_NA $>=3532.6, \mathrm{~N} \_B 1<=0.86$ ) $->$ yes & 0.60 \\
\hline
\end{tabular}

detection. Another concept that needs to be described is the $k$-factor rule, where $k$ is the number of risk factors. The rule here is a three-factor rule.

Based on the key RF, the procedure for the disease-rule generation is shown in Figure 10.
Combined with the disease-domain knowledge that is provided by the domain experts, the TPS has the power to ignore a large amount of the attributes at the very beginning, thereby leaving only the high risk factor support (RFS) attributes [15]. Among these attributes, two attribute sets are 
formed for a comparison based on the correlation; that is, if they are very similar to each other, they are strongly correlated, and the TPS will remove the one with the low RFS. Then, a basic association rule-mining algorithm like Apriori and commonly used decision-tree algorithms like C4.5, CART, or Random Forest will be used to generate the $k$-RF rules. For the first stage of the research, however, only C4.5 is used for the testing data. The algorithmic pseudocode is given in Algorithm 3. First, we calculate the GainRatio for each risk factor $r$ in $L$, then we choose the factor with the highest GainRatio and create a decision node based on this factor, split the dataset by this node. We repeat these steps until all nodes with appropriate satisfied GainRatio value have been used to generate the tree. The path from the root to leaf is the disease rule.

The reasons for the selection of $\mathrm{C} 4.5$ are its simplicity, the accuracy of its results, and the ability to use it on numerical and categorical attributes even with the presence of an overfitting problem.

During the next research stage, algorithms including CART, Random Forest, and KNN will be tested to find the one that fits the most datasets with a high accuracy. Finally, the generated rules will be stored in the HBase in preparation for the next few steps.

4.5. Disease Prediction and Detection. According to the disease rules and the RFS, the highly related key RFS, such as heavy drinking for hypertension, are used to generate the prediction model. This work was completed in the authors' previous study [15]. The multi-RFS will be compared with the disease rule to confirm the patient health condition, and again, this work was completed in [15].

4.6. Cloud-Service Module. The Cloud module plays the roles of data storage and transfer and consists of the public Cloud services GCSql and FCM. Considering the efficiency, connection, and security problems, only commonly used, important, and urgent information like an urgent message from the health-care provider is stored in the GCSql database. Alternatively, the FCM is salient for the communication between the TPS and any relevant device. Again, this work was completed in [15].

\section{Experiment}

At this stage, the detailed design work of the whole system has been finished, while the implementation work is partially finished. Further, the mobile health-sensor network has been set up in the experimental environment. A large amount of simulated data and a small number of real data that were downloaded from the Korea National Health and Nutrient Examination Survey (KNHANES) [24] were combined with simulated dataset, and the testing data comprises approximately 60,900 patient records, including basic personal information, disease information, and clinical information. The entire cluster has been established, and it can interact with Android devices through the Cloud module. Several devices have been used for the purpose of testing. Meanwhile, an

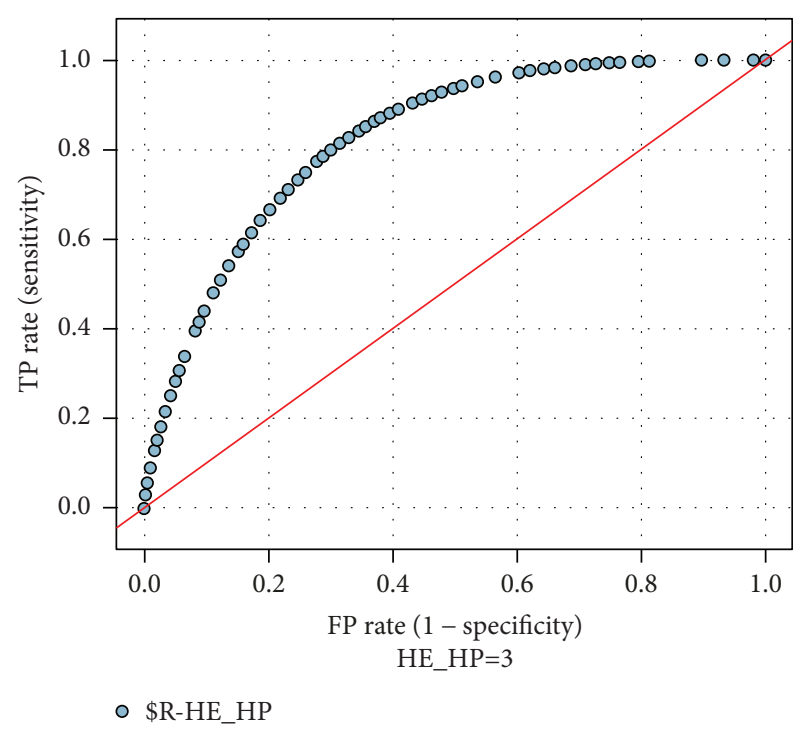

Figure 13: AUC of the trained SVM model. HE_HP $=3.0$ is the target attribute.

TABLE 6: Performance comparison of the two algorithms.

\begin{tabular}{lcccc}
\hline Method & Number of attr. & Sensitivity & Specificity & Accuracy \\
\hline C4.5 & 81 & 0.448 & 0.692 & 0.688 \\
SVM & 80 & 0.451 & 0.705 & 0.698 \\
\hline
\end{tabular}

app has been developed for the data collection and a dataset statistical-analysis-result visualization.

A MapReduce-based algorithm called "Disease Count" has been implemented and the pseudocode is given in Algorithm 1. The result of the Disease Count algorithm is given in Table 2. The hypertension data that contains 9383 records has been used as the test dataset, and its statistical-analysis results are given in Table 3. The number of main attributes is 3, but not all of the results are listed in this table due to a space limitation.

The advanced IoT device Fitbit Charge 2 has been used for user sports, sleeping, pulse, and breath detection. The model we used is shown in Figure 11.

Hybrid feature selection mechanism has been used to select highly related features. Among all these attributes, $t$ test and chi-squared test implemented by using $\mathrm{R}$ language have been applied to select the key RFS for hypertension. Finally, 217 features have been selected among all 526 features. The results are given in Figure 12.

SVM-based wrapper feature selection method has been applied to the attributes selected from the previous step. Therefore, 81 features have been selected and the top 8 features are given in Table 4 .

For the disease rule generation procedure, the minimum support threshold was set to 0.1 , and the minimum confidence threshold was set to 0.3 . C4.5 was used to generate the hypertension-disease rules that are shown in Table 5. DI1_dg $=0$ means hypertension not diagnosed, while DI1_dg = 1 means hypertension diagnosed. The result is given in Table 5. 


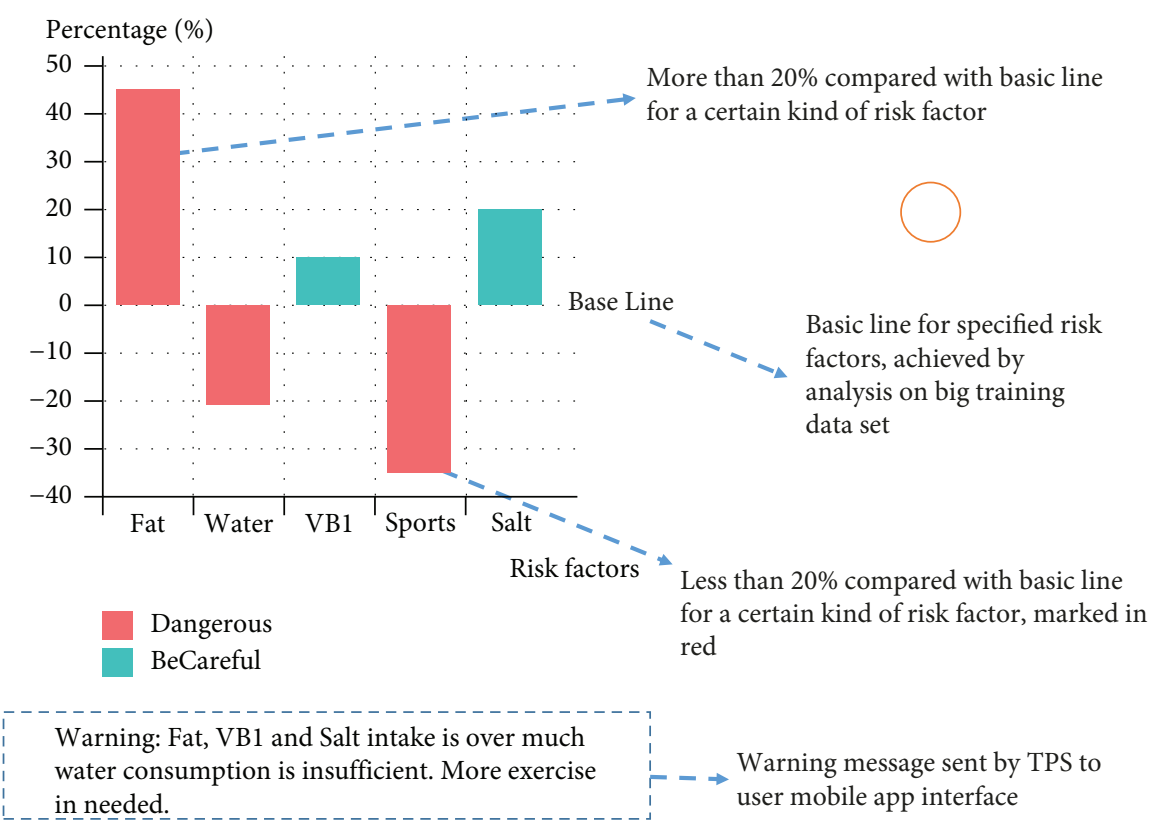

FIGURE 14: Rule with the risk factor (RF) analysis result visualization. The RFS marked in red mean too much or too few compared with the standard.

For the purpose of the comparison, SVM classification algorithm is also applied to predict the hypertension. We trained one model considered for the variable "HE_HP" for regressor following all the other 80 variables. The support vector machine results were implemented using the "e1071" package in $\mathrm{R}$ by performing a 10 -folder cross validation using radial basis kernel function which the best parameters are: $\operatorname{cost}=1000$, gamma $=0.01$. The result is given in Figure 13 .

We have compared the accuracy of the two methods in terms of sensitivity, specificity, and accuracy. The result is shown in Table 6 below,

From the results, it is possible to draw several conclusions as follows: (1) age and alcohol intake play very important role for hypertension; there is great chance for the elder and heavy drinkers having this disease. (2) The effect of smoking for hypertension is inferior to alcohol. (3) The elders should have light food instead of salty food. (4) SVM performs better result $\mathrm{C} 4.5$ in our dataset.

Nevertheless, the accuracy of both algorithms is not satisfactory. This is due to the challenges of collecting big healthcare data, and this issue has been depicted in introduction session. For the next stage of research, we will focus on solving this problem.

The analysis results are directly and visually displayed in the user devices. The interpretations of the analysis results are shown in Figure 14. The authors have published another paper [15] wherein a simple disease-rule visualization method is discussed, since it is also a challenging work.

Figure 14 illustrates the disease-detection-result visualization interface of the designed app of this study. The $x$-axis of the coordinate lists rank the key RFS of a certain kind of disease, and it is also the basic line that is based on the training big data analysis result (e.g., a standard factor like nutritional intake will be visible for a healthy patient, but the concrete value will be hidden from the figure). The $y$-axis is the percentage of the intake that exceeds or is inferior to the standard factor intake; the disease rules consist of these factors. For a certain disease, there is usually more than one rule (consisting of RFS) that is related to the disease. Compared with these rules, if the matching rate $>\beta$ (expertdefined threshold, e.g., $80 \%$ ), the system will treat this patient segment as the disease holder.

The figures below are GUI of our system, which is implemented based on Ionic using hybrid programming techniques. The characters are in Korean since it is mainly developed for Korean users so far. Selected user interfaces are given in Figure 15.

\section{Conclusion and Future Work}

In the present work, Hadoop, Spark, and DM techniques are exploited to design a comprehensive, real-time, and intelligent mobile health-care system that can facilitate a step-bystep process for disease detection and prediction. The purpose of this work is the provision of a practical assistant system for self-based user health care, as well as the design of a complementary system for patient disease diagnosis. During the experiment section, firstly, disease data stored in distributed environment has been retrieved by MapReduce method and analysed by statistical method to give us an overview of the data. Then both statistical methods and DM methods are used to select the features related to hypertension disease. These attributes are the risk factors as well. Based on these factors, C4.5 and SVM methods are used to generate the classifier model for disease prediction. Finally, we displayed the analysis result on users' mobile devices.

An overview and a guide are described in detail for a future work as well. For the next stage of research, after the implementation of the whole system, in-depth simulations will be performed to validate the systemic performance in 


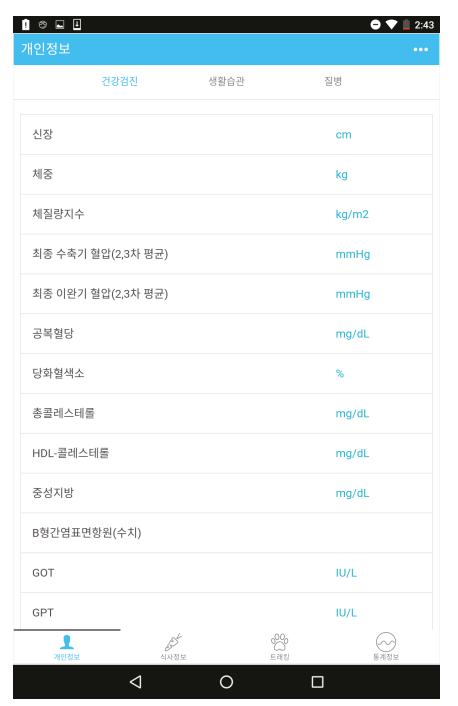

(a) User personal diagnostic information
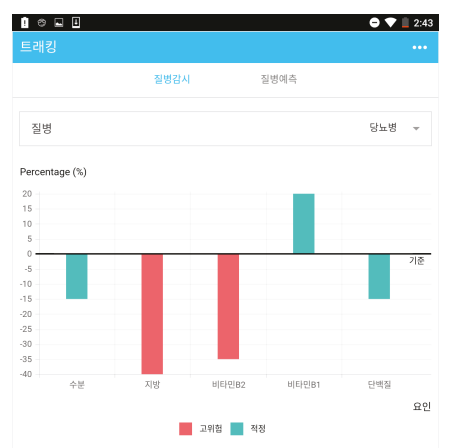

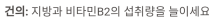

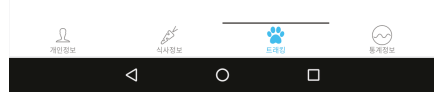

(c) Disease detection interface

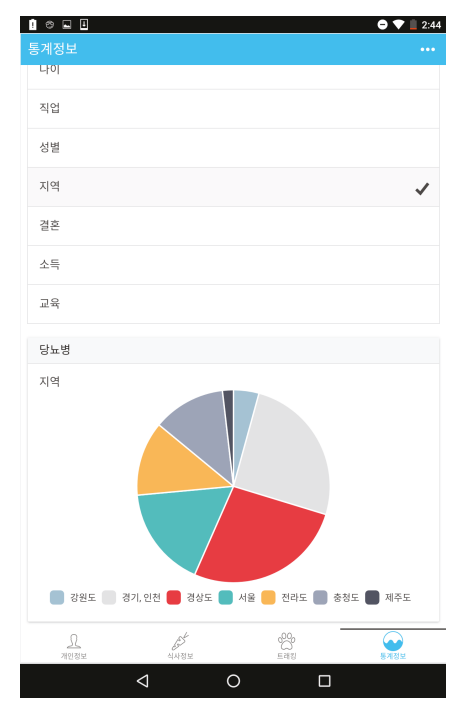

(b) Statistical analysis result

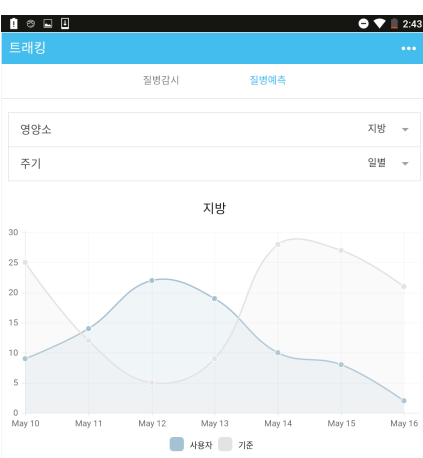

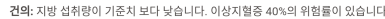

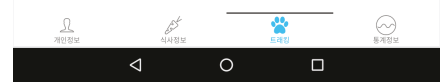

(d) Disease prediction interface

FIGURE 15: User interfaces of the system.

terms of the application of the proposed system in a real environment. The TPS and algorithms like C5, Random Forest, and other algorithms will be run on the TPS cluster to compare the efficiency and the accuracy. The procedure for the disease detection and prediction will be optimized continuously and extended to other chronic diseases. Finally, it is hoped that this system will contribute to health-care academia as well as the industry.

\section{Conflicts of Interest}

The authors declare that they have no conflicts of interest.

\section{Acknowledgments}

This work was supported by the Basic Science Research Program through the National Research Foundation of Korea (NRF) funded by the Ministry of Science, ICT \& Future Planning (Grant no.2017R1A2B4010826) and the MSIP
(Ministry of Science, ICT and Future Planning), Korea, under the ITRC (Information Technology Research Center) support program (IITP-2017-2013-0-00881), supervised by the IITP (Institute for Information \& Communication Technology Promotion) and also supported by the National Natural Science Foundation of China (61702324).

\section{References}

[1] A. V. Chobanian, G. L. Bakris, H. R. Black et al., "Seventh report of the joint national committee on prevention, detection, evaluation, and treatment of high blood pressure," Hypertension, vol. 42, no. 6, pp. 1206-1252, 2003.

[2] P. D. Kaur and I. Chana, "Cloud based intelligent system for delivering health care as a service," Computer Methods and Programs in Biomedicine, vol. 113, no. 1, pp. 346-359, 2014.

[3] H. Horiguchi, H. Yasunaga, H. Hashimoto, and K. Ohe, "A user-friendly tool to transform large scale administrative data into wide table format using a MapReduce program with a 
pig Latin based script," BMC Medical Informatics and Decision Making, vol. 12, no. 1, p. 151, 2012.

[4] R. C. Taylor, "An overview of the Hadoop/MapReduce/HBase framework and its current applications in bioinformatics," BMC Bioinformatics, vol. 11, article S1, Supplement 12, 2010.

[5] M. C. Schatz, "CloudBurst: highly sensitive read mapping with MapReduce," Bioinformatics, vol. 25, no. 11, pp. 1363-1369, 2009.

[6] V. Sharma, K. Mankodiya, F. De La Torre et al., "SPARK: personalized Parkinson disease interventions through synergy between a smartphone and a smartwatch," in Design, User Experience, and Usability. User Experience Design for Everyday Life Applications and Services. DUXU 2014, A. Marcus, Ed., vol. 8519 of Lecture Notes in Computer Science, pp. 103114, Springer, Cham, 2014.

[7] H. Kim, M. I. M. Ishag, M. Piao, T. Kwon, and K. H. Ryu, "A data mining approach for cardiovascular disease diagnosis using heart rate variability and images of carotid arteries," Symmetry, vol. 8, no. 6, p. 47, 2016.

[8] S. Amendola, R. Lodato, S. Manzari, C. Occhiuzzi, and G. Marrocco, "RFID technology for IoT-based personal healthcare in smart spaces," IEEE Internet of Things Journal, vol. 1, no. 2, pp. 144-152, 2014.

[9] M. J. Huang, M. Y. Chen, and S. C. Lee, "Integrating data mining with case-based reasoning for chronic diseases prognosis and diagnosis," Expert Systems with Applications, vol. 32, no. 3, pp. 856-867, 2007.

[10] S. H. Ha and S. H. Joo, "A hybrid data mining method for the medical classification of chest pain," International Journal of Computer and Information Engineering, vol. 4, no. 1, pp. 3338, 2010.

[11] H. J. Aboumatar, K. A. Carson, M. C. Beach, D. L. Roter, and L. A. Cooper, "The impact of health literacy on desire for participation in healthcare, medical visit communication, and patient reported outcomes among patients with hypertension," Journal of General Internal Medicine, vol. 28, no. 11, pp. 1469-1476, 2013.

[12] Y. Piao, M. Piao, C. H. Jin et al., "A new ensemble method with feature space partitioning for high-dimensional data classification," Mathematical Problems in Engineering, vol. 2015, Article ID 590678, 12 pages, 2015.

[13] M. Herland, T. M. Khoshgoftaar, and R. Wald, "Survey of clinical data mining applications on big data in health informatics," in 2013 12th International Conference on Machine Learning and Applications, vol. 2, pp. 465-472, Miami, FL, USA, December 2013.

[14] R. Fang, S. Pouyanfar, Y. Yang, S. C. Chen, and S. S. Iyengar, "Computational health informatics in the big data age: a survey," ACM Computing Surveys (CSUR), vol. 49, no. 1, article 12, 2016.

[15] D. Li, H. Park, E. Batbaatar, Y. Piao, and K. Ryu, "Design and partial implementation of health care system for disease detection and behavior analysis by using DM techniques," in 2016 IEEE 14th Intl Conf on Dependable, Autonomic and Secure Computing, 14th Intl Conf on Pervasive Intelligence and Computing, 2nd Intl Conf on Big Data Intelligence and Computing and Cyber Science and Technology Congress(DASC/PiCom/DataCom/CyberSciTech), pp. 781-786, Auckland, New Zealand, August 2016.

[16] "Welcome to Apache ${ }^{\mathrm{TM}}$ Hadoop," http://hadoop.apache.org/.
[17] "Apache ${ }^{\mathrm{TM}}$ Spark, a fast and general engine for large-scale data processing," http://spark.apache.org/.

[18] J. Han, J. Pei, and M. Kamber, Chapter 1 Introduction Data Mining: Concepts and Techniques, Elsevier, Waltham, MA, USA, 2011.

[19] C. Cortes and V. Vapnik, "Support-vector networks," Machine Learning, vol. 20, no. 3, pp. 273-297, 1995.

[20] H. H. Hsu, C. W. Hsieh, and M. D. Lu, "Hybrid feature selection by combining filters and wrappers," Expert Systems with Applications, vol. 38, no. 7, pp. 8144-8150, 2011.

[21] “Apache Sqoop ${ }^{\mathrm{TM}}$," http://sqoop.apache.org/.

[22] B. Lee and E. Jeong, "A design of a patient-customized healthcare system based on the hadoop with text mining (PHSHT) for an efficient disease management and prediction," International Journal of Software Engineering and Its Applications, vol. 8, no. 8, pp. 131-150, 2014.

[23] “Apache Spark ${ }^{\mathrm{TM}}$," http://spark.apache.org/streaming/.

[24] "KNHANES," https://knhanes.cdc.go.kr/knhanes/sub03/sub 03_02_02.do. 


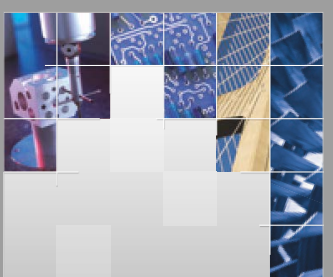

\section{Enfincering}
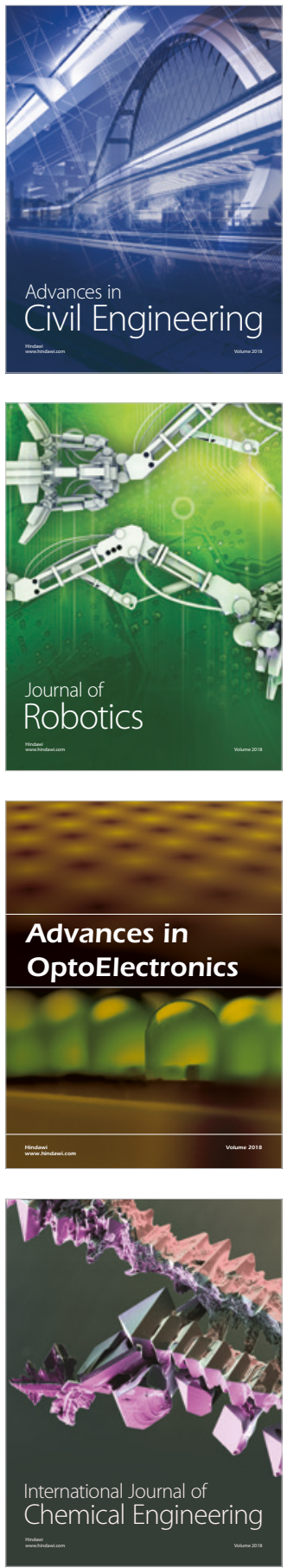

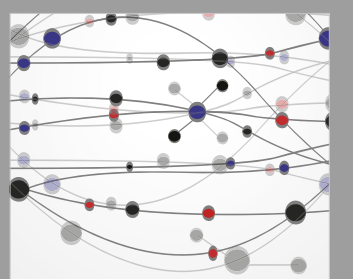

\section{Rotating \\ Machinery}

The Scientific World Journal

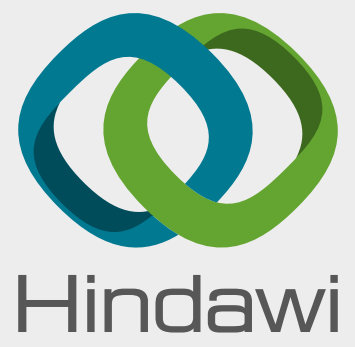

Submit your manuscripts at

www.hindawi.com
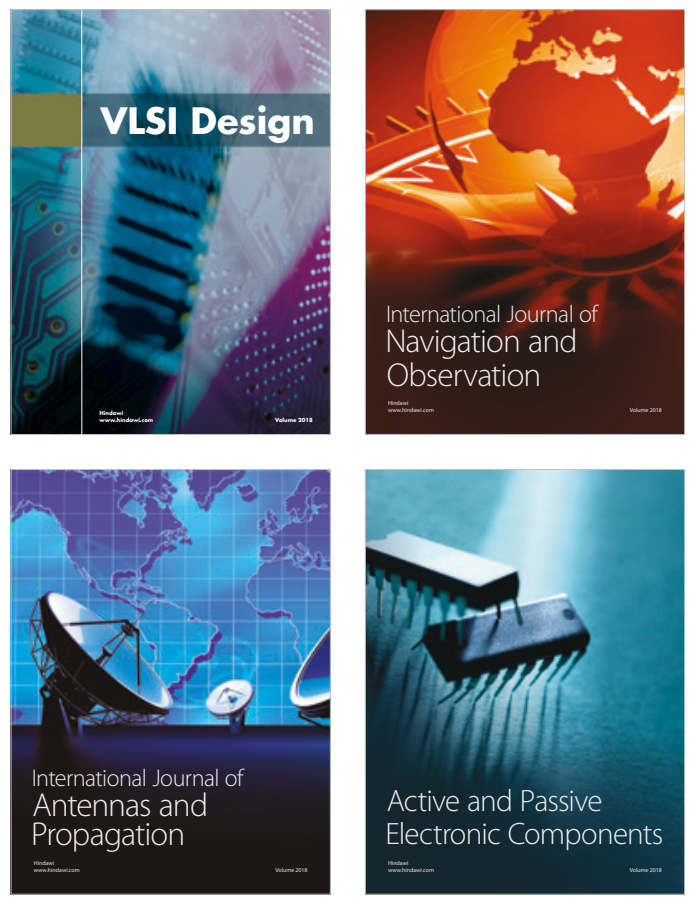
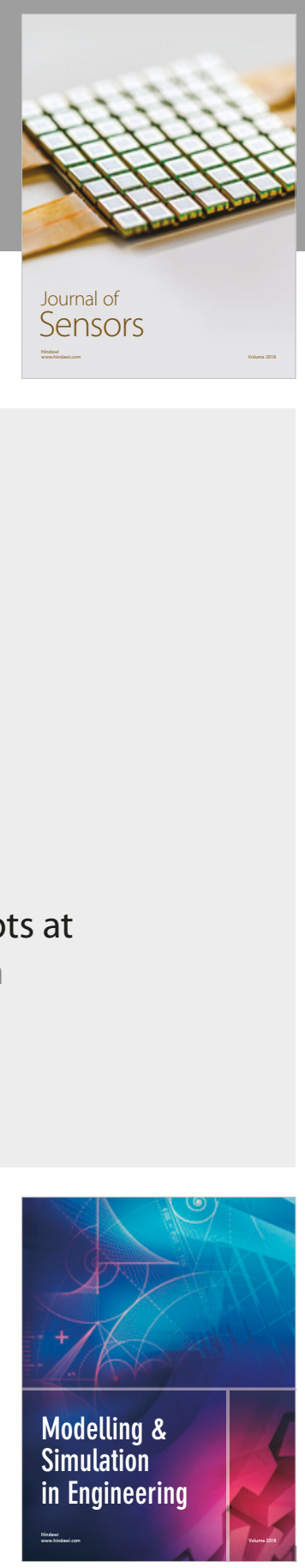

\section{Advances \\ Multimedia}
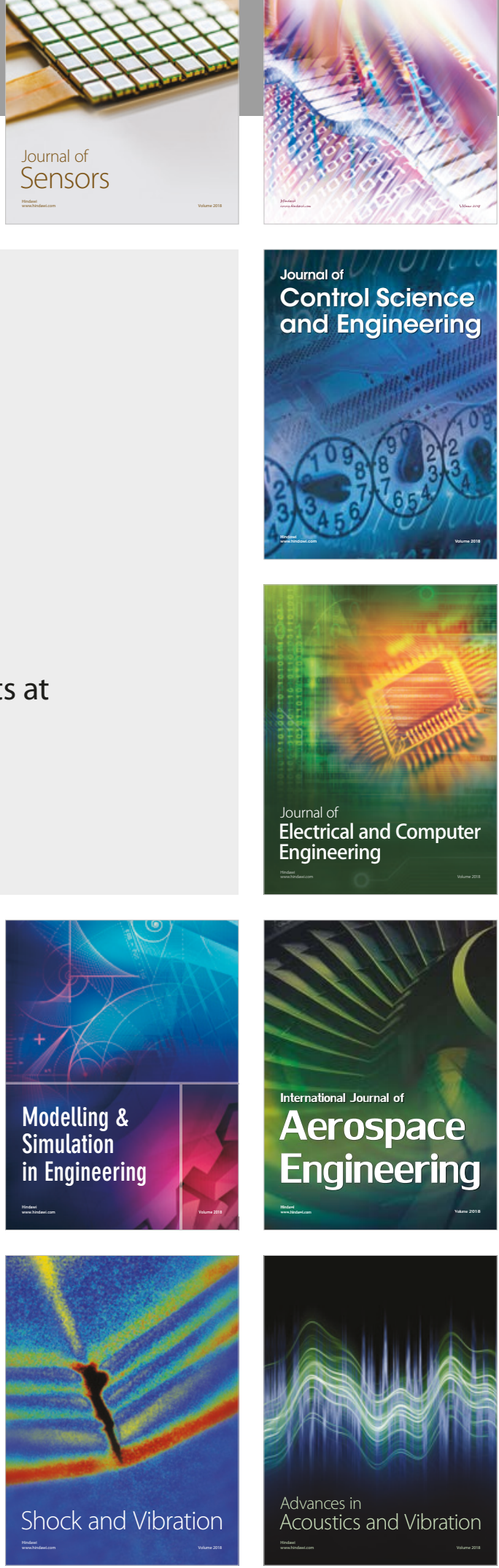